\title{
Effects of a Cav2.2 inhibitor on spatial and non-spatial short-term memory
}

\author{
Ying Zhou ${ }^{1}$, Kimie Niimi ${ }^{2}$, Weidong Li $^{1}$, and Eiki Takahashi, ${ }^{1, *}$ \\ ${ }^{1}$ Bio-X Institutes, Key Laboratory for the Genetics of Developmental and Neuropsychiatric Disorders (Ministry of Education), Shanghai Jiao Tong University, \\ Shanghai, 200240, People's Republic of China \\ ${ }^{2}$ Research Resources Center, RIKEN Brain Science Institute, Saitama, 351-0198, Japan
}

\begin{abstract}
Neuronal voltage-gated calcium channels (VGCCs), including Cav2.1 and Cav2.2, mediate the presynaptic machinery responsible for vesicular release of neurotransmitters. However, the role of different VGCCs in neural circuits underlying spatial and non-spatial short-term memory remains poorly understood. Previous report has shown that Cav2.1-mediated signaling is required on spatial memory in a hippocampus-related object location test but has little effect on nonspatial memory in a perirhinal cortex-related object recognition test. Thus, Cav2.2-mediated functions need to be clarified. This study examined whether Cav2.2mediated signaling plays a role in spatial and non-spatial short-term memory. Intracerebroventricular injection of the Cav2.2 inhibitor $\omega$-conotoxin GVIA (5 pg/ side) in mice resulted in deficits in object location and recognition tests indicative of short-term memory. These results indicate that different VGCCs have different effects on memory performance.
\end{abstract}

\section{Introduction}

Voltage-gated calcium channels (VGCCs) mediate a number of neuronal functions including neurotransmitter release, neuronal excitation, neurite outgrowth, synaptogenesis, neuronal survival, differentiation and plasticity, as well as regulate gene expression [1$3]$. VGCCs are molecular complexes comprised of $\alpha 1, \beta, \alpha 2-\delta$, and $\gamma$ subunits [1]. The $\alpha 1$ subunit is essential for channel function and determines fundamental channel properties [1]. Genes encoding 10 pore-forming $\alpha 1$ subunits and several splice variants have been identified and characterized [4].

The role of different VGCCs in the neural circuits underlying spatial and non-spatial short-term memory has not been investigated in detail. Previous reports indicated that short-term memory required Cav2.1-mediated signaling in a hippocampus-related object location test but had little effect on non-spatial memory in a perirhinal cortexrelated object recognition test [5]. Thus, Cav2.2-mediated functions need to be clarified

Several types of behavioral tasks have been designed to examine memory formation in rodents [6]. Most of these tasks use positive or negative reinforcement, such as water immersion for the water maze test and electrical stimulation for the fear-conditioning test. In these tests, animals learn a rule through reinforcement during the training phase; however, human cognitive performance is not normally tested with reinforcers, supporting the use of cognitive tests without reinforcers as more appropriate for investigating the relationship between VGCC function and memory formation in humans.

The object location test is based on the spontaneous tendency of rodents to explore an object placed in a novel location for a longer period of time than that of a non-displaced identical object or more frequently than that of a familiar one $[7,8]$. Both tests have been used to evaluate memory without the need for conventional reinforcers. Neuronal VGCCs, including Cav2.1 and Cav2.2, are predominantly expressed at presynaptic neuronal terminals throughout the central nervous systems [9].

Previous studies have indicated that the hippocampus and perirhinal cortex are involved in object location memory and object recognition memory, respectively [10-13]. In this study, we examined whether Cav2.2-mediated signaling plays a role in spatial and nonspatial short-term memory by conducting an object location test for spatial memory and an object recognition test for non-spatial memory using mice treated with the Cav2.2 blocker, $\omega$-conotoxin GVIA.

\section{Materials and methods}

\section{Mice}

All animal procedures were approved by the Animal Experiments Committee of Shanghai Jiao Tong University and RIKEN. C57BL/6J mice were provided by Charles River Japan (Kanagawa, Japan). The mice were given free access to water and food pellets (CRF-1; Oriental Yeast Co. Ltd., Tokyo, Japan) and housed under a 12/12-h light/dark cycle (lights on from 08:00 to 20:00) at $23 \pm 1^{\circ} \mathrm{C}$ and $55 \pm 5 \%$ humidity. We used separate groups of 2-month-old male mice for each of the in situ hybridization (ISH) behavioral tests. All experiments were conducted by investigators blinded to the treatment conditions.

Correspondence to: Eiki Takahashi, DVM, PhD, Research Resources Center RIKEN Brain Science Institute, 2-1 Hirosawa, Wako, Saitama, 351-0198, Japan, Tel: +81-48-467-5871; Fax: +81-48-467-9692; E-mail: etakahashi@brain.riken.jp

Key words: Cav2.2, object location test, object recognition test, $\omega$-conotoxin GIVA, short-term memory

Received: July 20, 2015; Accepted: August 18, 2015; Published: August 22, 2015 


\section{In situ hybridization}

At the end of the experiment, the brains were dissected after perfusion, fixed in tissue fixative (Gonostaff, Tokyo, Japan), embedded in paraffin, and sectioned (6- $\mu \mathrm{m}$-thick slices). Hybridization was conducted as reported previously [14] using a 691-bp cDNA fragment corresponding to 6068-6748 bp of Cav2.1 $\alpha 1$ cDNA (Accession Number: NM_007578.3).

\section{Infusion}

For the infusion studies, the Cav2.2 blocker, $\omega$-conotoxin GVIA (100 pg/ $\mu \mathrm{L}$, Peptide Institute, Osaka, Japan) was dissolved in saline (vehicle). The drug dose was determined based on a previous report $[15,16]$. Non-treated mice received an equivalent volume of vehicle. Under anesthesia and using standard stereotaxic procedures, stainlesssteel guide cannulae (22-gauge) were implanted into the lateral ventricle (posterior to bregma, $-0.34 \mathrm{~mm}$; lateral to midline, $\pm 0.9 \mathrm{~mm}$; ventral from the dura, $-2.3 \mathrm{~mm}$ ), and mice were allowed to recover for at least 1 week following surgery. The mice were briefly anesthetized with isoflurane to facilitate insertion of the injection cannula (26-gauge). Infusion into the lateral ventricle $(0.1 \mu \mathrm{L} /$ side $)$ was accomplished at a rate of $0.05 \mu \mathrm{L} / \mathrm{min} 30 \mathrm{~min}$ before behavioral testing. The injection cannula was left in place for $2 \mathrm{~min}$ following infusion.

\section{Behavioral test}

Mice were moved into the behavioral testing room at least $2 \mathrm{~h}$ prior to testing. The object location and object recognition tests were performed as reported with slight modifications [7,8]. Both tests consisted of three phases: habituation, acquisition, and retention (30 min after the acquisition phase) conducted within a polycarbonate cage $(35 \times 35 \times 35 \mathrm{~cm})$ under illumination of 25 lux. Procedures for the habituation and acquisition phases were identical for the object recognition and object location tests. During the habituation phase, mice were individually subjected to a single familiarization session of $60 \mathrm{~min}$ each day for 3 days, during which they were introduced to the empty arena to familiarize them with the apparatus.

During the acquisition phase, mice were subjected to a single 5-min session, during which two identical floor-fixed plastic columns $(4 \mathrm{~cm}$ high $\times 5 \mathrm{~cm}$ diameter) (A) were placed symmetrically in the center of the arena, $11 \mathrm{~cm}$ from each other and $12 \mathrm{~cm}$ from the nearest wall. Mice were allowed to explore the objects in the open field. To minimize the presence of olfactory trails, the objects were cleaned thoroughly with $70 \%$ ethanol before each trial. During the retention phase of the object location test, mice were allowed to explore the open field in the presence of two identical objects: non-displaced object A and displaced object $B$ (placed in the corner of the apparatus) for $10 \mathrm{~min}$. The displacement location was counterbalanced in the group. The time spent exploring each object (nose pointing toward the object at a distance of $<1 \mathrm{~cm}$ ) was recorded by hand. The recognition index (i.e., the ratio of the amount of time spent exploring either object [acquisition phase] or the displaced object in particular [retention phase] divided by the total time spent exploring both objects) was used to measure spatial memory performance.

For the object recognition test, the mice were allowed to explore the open field for $10 \mathrm{~min}$ in the presence of two different objects during the retention phase: the familiar object $\mathrm{A}$ and a novel object $\mathrm{B}$ (triangular pyramid: $4 \mathrm{~cm}$ high $\times 5 \mathrm{~cm}$ base area). The starting object and object pairs were counterbalanced in the group. The time spent exploring the object (nose pointing toward the object at a distance $<1$ $\mathrm{cm}$ ) was recorded by hand. The recognition index (i.e., the ratio of the amount of time spent exploring any object [acquisition phase] or the novel object in particular [retention phase] divided by the total time spent exploring both objects) was used to measure non-spatial shortterm memory.

Data are presented as means \pm standard error of the mean (SEM). Statistical analyses for the object location and object recognition tests were conducted using Excel Statistics 2006 (SSRI, Tokyo, Japan). Data were analyzed by repeated measures ANOVA with Tukey's test.

\section{Histology}

Histological verification of the cannula locations was performed at the end of behavioral testing. Mice were perfused transcardially with $0.9 \%$ saline, followed by $4 \%$ paraformaldehyde (PFA). After extraction from the skull, the brains were post-fixed in $4 \%$ PFA and then transferred to a $30 \%$ sucrose solution until sectioning. Coronal sections $(40 \mu \mathrm{m}$ thick, taken every $120 \mu \mathrm{m})$ were cut on a cryostat $\left(-16^{\circ} \mathrm{C}\right)$ and mounted on glass microscope slides. After drying, the sections were stained with cresyl violet.

\section{Results}

We used ISH to determine the Cav2.2a1 expression patterns in mouse brains. We performed the object location test for spatial memory and the object recognition test for non-spatial memory. During the acquisition phase, the mice were individually allowed to explore two objects freely to enable familiarization, and during the retention phase, we assessed the memories of the trained mice.

\section{Expression of Cav2.2a1 mRNA}

Using the antisense probe, widespread expression of the $\alpha_{1}$ subunit of Cav2.2 was detected in the brain, with particularly high expression levels evident in the hippocampus and perirhinal cortex (Figure 1). The sense probe yielded no signal from any of the brains (data not shown).

\section{Intracerebroventricular injection effects of $\omega$-conotoxin GVIA on spatial short-term memory in the object location test}

The object location test used male mice given intracerebroventricular injections of 0 (vehicle) or $10 \mathrm{pg} /$ side $\omega$-conotoxin GVIA $(n=10$ each) (Figure 2). The exploration times in the acquisition phase for vehicle-injected and $\omega$-conotoxin GVIA-injected mice were $151.3 \pm$ 8.96 and $153.8 \pm 9.79 \mathrm{sec}$, respectively. During the training session, no significant differences were found between vehicle-injected and

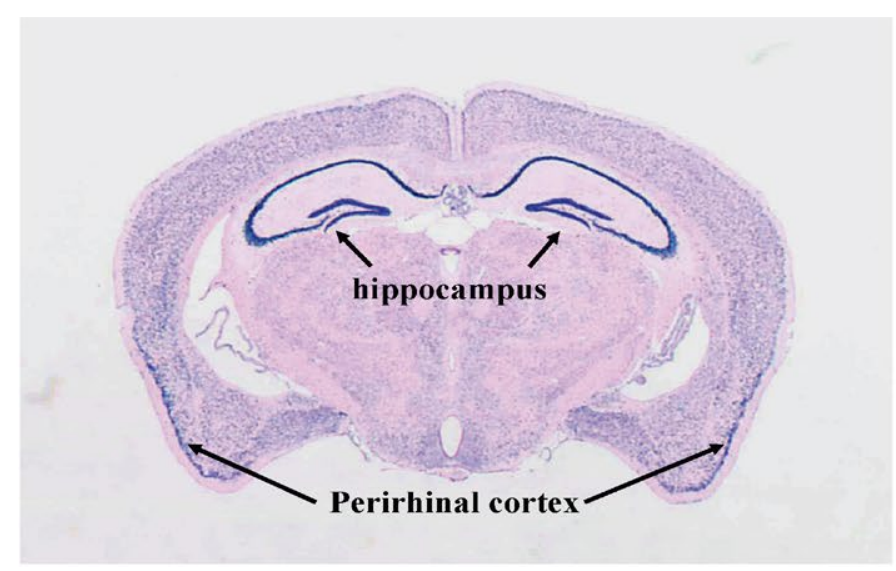

Figure 1. In situ hybridization for Cav2.1 11 mRNA. 


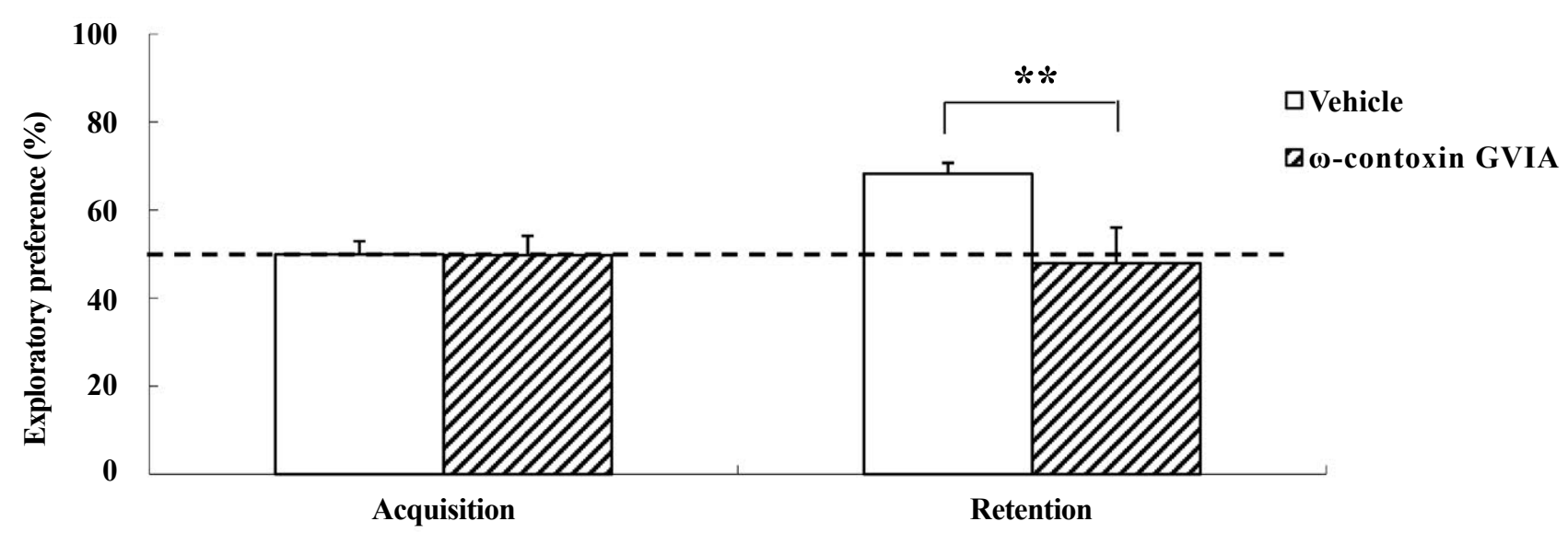

Figure 2. The effects of intracerebroventricular injection of $\omega$-conotoxin GVIA on the object location test. During the retention phase, a significantly stronger exploratory preference was detected for the displaced object than for the non-displaced object in vehicle-injected mice. No significant differences were found in the time spent between the displaced and non-displaced objects in $\omega$-conotoxin GVIA-injected mice. The data are presented as means \pm standard error of the mean (SEM). $* * P<0.01$ compared with the appropriate control (Tukey's test).

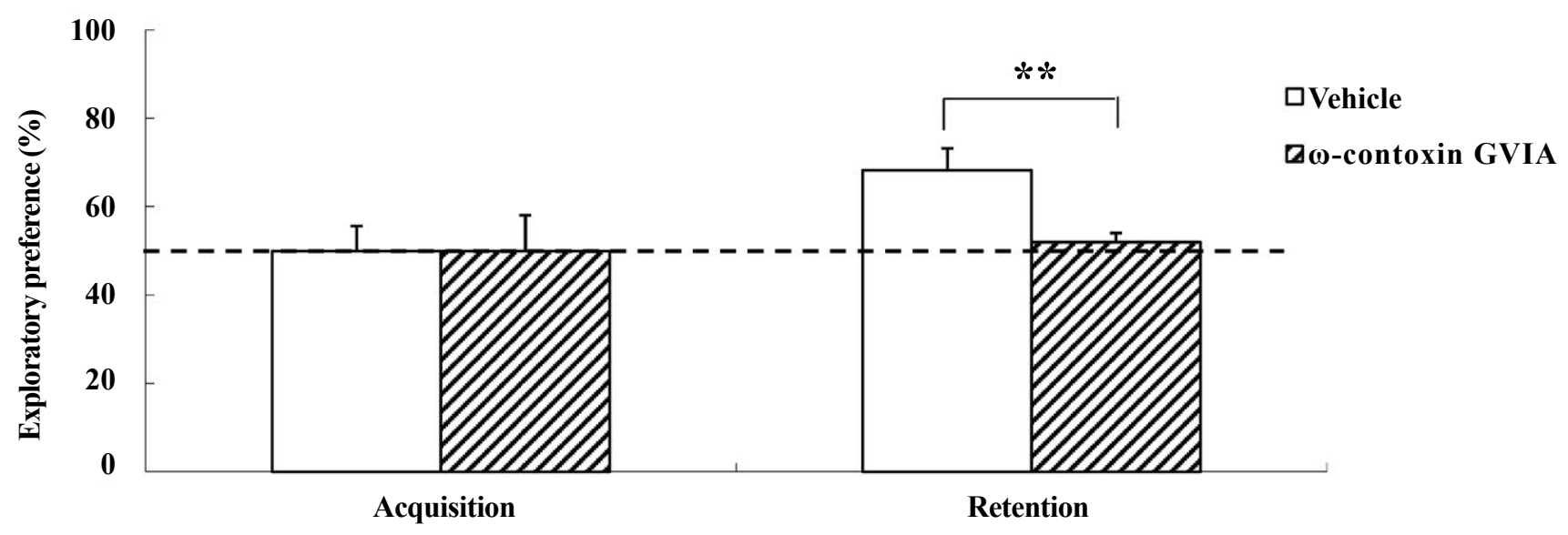

Figure 3. The effects of intracerebroventricular injection of $\omega$-conotoxin GVIA on the object recognition test. During the retention phase, a significantly stronger exploratory preference was detected for the novel object than for the familiar object in vehicle-injected mice. No significant differences were found in the time spent between objects in $\omega$-conotoxin GVIA-injected mice. The data are presented as means \pm standard error of the mean (SEM). $* * P<0.01$ compared with the appropriate control (Tukey's test).

$\omega$-conotoxin GVIA-injected mice in terms of the time spent exploring the two objects. After $30 \mathrm{~min}$, we evaluated the trained mice for memory. The exploration times in the retention phase for vehicleinjected and $\omega$-conotoxin GVIA-injected mice were $411.5 \pm 15.49$ and $301.2 \pm 8.13 \mathrm{sec}$, respectively. Although the $\omega$-conotoxin GVIAinjected mice spent an equal amount of time exploring the two objects during the exploration sessions, vehicle-injected mice showed an exploratory preference for the displaced object. Mice with injection needle placements outside of the boundaries of the targeted areas were excluded from the behavioral analysis (data not shown). These results show that blockade of Cav2.2-mediated signaling impairs spatial shortterm memory.

Intracerebroventricular injection effects of $\omega$-conotoxin GVIA on non-spatial short-term memory in the object recognition test

The object recognition test was performed on male mice given cerebroventricular injections of 0 (vehicle) or $10 \mathrm{pg} / \mathrm{side} \omega$-conotoxin
GVIA ( $n=10$ each). In each test (Figure 3 ), the exploration times in the acquisition phase for vehicle-injected and $\omega$-conotoxin GVIAinjected mice were $153.0 \pm 8.11$ and $149.0 \pm 10.26 \mathrm{sec}$, respectively. We found no significant differences between vehicle-injected and $\omega$-conotoxin GVIA-injected mice in terms of time spent exploring the two objects during the training session. After $30 \mathrm{~min}$, we evaluated the trained mice for memory. The exploration times for vehicle-injected and $\omega$-conotoxin GVIA-injected mice were $414.5 \pm 12.56$ and 302.0 $\pm 15.21 \mathrm{sec}$, respectively. Although vehicle-injected mice showed an exploratory preference for the novel object, $\omega$-conotoxin GVIA-injected mice showed similar time spent exploring the two objects. Mice with injection needle placements outside of the boundaries of the targeted areas were excluded from behavioral analysis (data not shown). These results shows that blockade of Cav2.2-mediated signaling impairs nonspatial short-term memory.

\section{Discussion}

Neuronal VGCCs, including Cav2.1 and Cav2.2, mediates 
neurotransmitter release from presynaptic machinery that underlies behavior. However, the role of different VGCCs in the neural circuits underlying spatial and non-spatial short-term memory has not been explored. In the present study, we investigated the relationship between Cav2.2-mediated signaling and spatial and non-spatial shortterm memory formation in mice that received intracerebroventricular injection of the Cav2.2 blocker $\omega$-conotoxin GVIA.

We first examined the expression pattern of Cav2.2a1 in the brain. ISH revealed that Cav2.2 $\alpha 1$ was predominantly expressed throughout the central nervous system, including the hippocampus and perirhinal cortex, as reported previously [9]. Given that precise regulation of $\mathrm{Ca}^{2+}$ signaling is important for neuronal processes, alterations in the $\mathrm{Ca}^{2+}$ current through different Cav2 channels would affect the functioning of neurons and circuits that lead to memory formation. It has been reported that Cav2.1a1 is strongly expressed in the hippocampus and perirhinal cortex [17]. Our previous study examined whether a subtle disruption of Cav2.1 channel functioning is sufficient to cause deficits in memory formation using the hippocampus-related object location test for spatial memory and perirhinal cortex-related object recognition test for non-spatial memory in aged heterozygous Rolling Nagoya (rol/+) mice carrying a Cav2.1a1 mutation [5]. During the acquisition phase, mice were individually allowed to explore two objects freely to enable familiarization. The procedures used in the acquisition phase of the object location and object recognition tests are identical, indicating that the same information including location and characteristics about the two identical objects, as well as the extra-field cues, would be encoded during the acquisition phase of both tests. The $\mathrm{rol} /+$ mice displayed a normal acquisition phase of both tests in terms of the time spent exploring the two objects, indicating that the mice had the same levels of curiosity and motivation for the task. During the retention phase, we tested the trained mice for memory as the present study. The performances of $\mathrm{rol} /+$ mice during the exploration sessions showed memory deficits in the object location but not the object recognition test. These results indicate that mice recognized the new location or the novel object by means of different kinds of neuronal processes with Cav2.1 channels in different regions. On the other hand, although there were no significant differences between vehicle-injected and $\omega$-conotoxin GVIA-injected mice in terms of time spent exploring the two objects during the acquisition phase of the object location and object recognition tests, the performances of $\omega$-conotoxin GVIAinjected mice during the retention phase indicated memory deficits in the object location as well as object recognition test, suggesting that the signal cascade activated by Cav2.2 plays a role in hippocampusdependent spatial and perirhinal cortex-dependent non-spatial shortterm memory.

In conclusion, we found that a Cav2.2 blocker disrupted the spatial and non-spatial short-term memory in the object location and object recognition tests. To examine how Cav2.1 or Cav2.2 channel-dependent signaling influence short-term memory in the hippocampus and perirhinal cortex, electrophysiological studies and neurotransmitter release analyses will be required. We have also showed that the combination of different sub-threshold doses of agents and local infusions are useful for inducing the effects of short-term learning and for identifying functional signaling pathways in specific neuronal circuits [18]. Thus, subsequent studies should examine the relationship between Cav2-mediated signaling and short-term memory in a similar combination study.

\section{Acknowledgments}

This work was supported by China 973 project (2010CB529604) and National Scientific Foundation of China (81271511 and 30900432).

\section{Conflicts of interest}

The authors declare no competing interests.

\section{Authors' contributions}

WL and ET designed and supervised the research, and wrote the manuscript. YZ and KN performed the surgeries and behavioral experiments. All authors read and approved the final version of the manuscript.

\section{References}

1. Catterall WA, Few AP (2008) Calcium channel regulation and presynaptic plasticity. Neuron 59: 882-901. [Crossref]

2. Evans RM, Zamponi GW (2006) Presynaptic Ca2+ channels--integration centers for neuronal signaling pathways. Trends Neurosci 29: 617-624. [Crossref]

3. Jarvis SE, Zamponi GW (2007) Trafficking and regulation of neuronal voltage-gated calcium channels. Curr Opin Cell Biol 19: 474-482. [Crossref]

4. Takahashi E (2012) Cav2.1 channelopathy and mouse genetic approaches to investigate function and dysfunction of Cav2.1 channel. In: M. Yamaguchi, ed. Calcium Signaling. New York. Nova Science Publishers, Inc. Chapter 7. p149-158.

5. Takahashi E, Niimi K, Itakura C (2009) Age-related spatial and nonspatial short-term memory in Cav2.1alpha1 mutant mice, Rolling Nagoya. Behav Brain Res 204: 241245. [Crossref]

6. Crawley JN (1992) Learning and Memory. In: JN. Crawley, ed. What's wrong with my mouse?: Behavioral phenotyping of transgenic and knockout mice. New York. WileyLiss. Chapter 6. p86-130.

7. Murai T, Okuda S, Tanaka T, Ohta H (2007) Characteristics of object location memory in mice: Behavioral and pharmacological studies. Physiol Behav 90: 116-124. [Crossref]

8. Niimi K, Takahashi E, Itakura C (2008) Improved short-term memory and increased expression of NR2B observed in senescence-accelerated mouse (SAM) P6. Exp Gerontol 43: 847-852. [Crossref]

9. Tanaka O, Sakagami H, Kondo H (1995) Localization of mRNAs of voltage-dependent $\mathrm{Ca}(2+)$-channels: four subtypes of alpha 1- and beta-subunits in developing and mature rat brain. Brain Res Mol Brain Res 30: 1-16. [Crossref]

10. Bussey TJ, Duck J, Muir JL, Aggleton JP (2000) Distinct patterns of behavioura impairments resulting from fornix transection or neurotoxic lesions of the perirhinal and postrhinal cortices in the rat. Behav Brain Res 111: 187-202. [Crossref]

11. Ennaceur A, Neave N, Aggleton JP (1997) Spontaneous object recognition and object location memory in rats: the effects of lesions in the cingulate cortices, the medial prefrontal cortex, the cingulum bundle and the fornix. Exp Brain Res 113: 509-519. [Crossref]

12. Murray EA, Richmond BJ (2001) Role of perirhinal cortex in object perception, memory, and associations. Curr Opin Neurobiol 11: 188-193. [Crossref]

13. Winters BD, Bussey TJ (2005) Glutamate receptors in perirhinal cortex mediate encoding, retrieval, and consolidation of object recognition memory. $J$ Neurosci 25 : 4243-4251. [Crossref]

14. Sakuraoka Y, Sawada T, Shiraki T, Park K, Sakurai Y, et al. (2012) Analysis of hepcidin expression: in situ hybridization and quantitative polymerase chain reaction from paraffin sections. World J Gastroenterol 18: 3727-3731. [Crossref]

15. Zhou Y, Niimi K, Li W, Takahashi E (2015) Spatial short-term memory regulated by Cav2.2-mediated NMDA receptor signaling. Integr Mol Med 2: 131-134.

16. Zhou Y, Niimi K, Li W, Takahashi E (2015) Disruption of spatial cognition by intraaccumbens injection of Cav2.2 inhibitor. Integr Mol Med 2: 109-111. 
17. Fletcher CF, Lutz CM, O’Sullivan TN, Shaughnessy JD Jr, Hawkes R, et al. (1996) Absence epilepsy in tottering mutant mice is associated with calcium channel defects. Cell 87: 607-617. [Crossref]
18. Takahashi E, Niimi K, Itakura C (2010) Impairment of spatial short-term memory following acute administration of the NMDA receptor antagonist in heterozygous rolling Nagoya mice carrying the Cav2.1 alphal mutation. Behav Brain Res 213: $121-125$.

Copyright: (C2015 Zhou Y. This is an open-access article distributed under the terms of the Creative Commons Attribution License, which permits unrestricted use, distribution, and reproduction in any medium, provided the original author and source are credited. 